\author{
MILITARY TECHNICAL COLLEGE \\ CAIRO - EGYPT
}

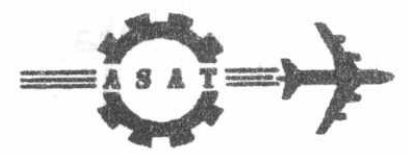
$7^{\text {th }}$ INTERNATIONAL CONF. ON AEROSPACE SCIENCES \& AVIATION TECHNOLOGY

\title{
Computational Solution of Aircraft Stability Derivatives
}

\author{
$\begin{array}{lll}\text { M. I. I. Mostafa* } & \text { M. H. Katary** } & \text { O. E. Abd El-Hamid*** }\end{array}$
}

\begin{abstract}
The present work describes a method for calculating inviscid, incompressible and steady flows around an aircraft. The proposed method is based upon a combination of the surface source panel method for the body, and the vortex lattice method for the lifting surfaces and their separated flows. The configuration as a whole is treated in a single global three-dimensional system of equations. Solving this three-dimensional system of equations we can get the strengths of the singularities, hence, the pressure distribution on the configuration surfaces and the resultant aerodynamic properties. Experimental work is made in M.T.C. wind tunnel on a jet trainer aircraft model .In order to demonstrate the capability of the present method to predict the pressure distribution on the configuration surface and hence explain the aerodynamic phenomena of wing-body-tail combinations, comparisons are made with the experimental results. These results demonstrate the same general trends obtained from calculations.
\end{abstract}

\section{Introduction}

The present work deals with the determination of the longitudinal aerodynamic characteristics of the aircraft as a three dimensional configuration. Here we calculate some of the longitudinal stability derivatives for a wing-body-tail configuration including the interference effects. A variery of the available methods for the determination of the above mentioned problem are briefly reviewed. Also methods dealing with the interference effects between the individual parts of the configuration. From the different methods of solution, we chose three methods for solving the above mentioned problems. These methods are Vortex Lattice Method for solving isoiated and multi-lifting surfaces. Source Panel Method for the solution of the bodies. Finally, a combination of the two mentioned methods for the calculation of the complete aircraft in a global system of equation. It may be stated that the present method can serve as a useful tool for the investigation of the flow patterns around various flight vehicles, and for the calculation of their aerodynamic properties during preliminary design.

* Engineer, R\&D Center For Aviation Systems, E.A.F.

** Assistant Professor, R\&D Center For Aviation Systems, E.A.F.

*** Professor, Department of Aeronautics, M.T.C.

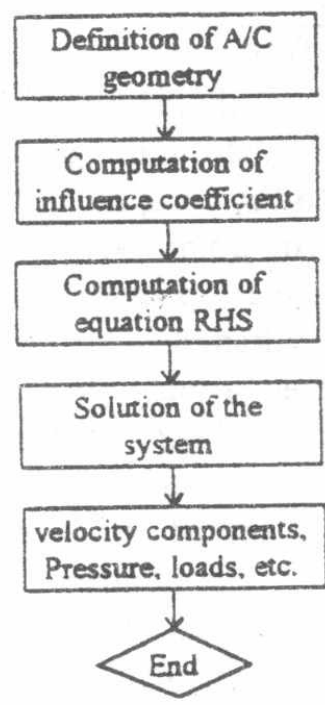

Fig. 1. Schematic flow chart for the numerical solution of the surface singularity distribution problem 


\section{Mathematical Model}

The application of the numerical techniques allows the treatment of more realistic geometry, and the fulfillment of the boundary conditions on the actual surface. The methods presented here are based on the surface distribution of singularity elements. It is very important to realize that the grid dose have an effect on the solution. Typically, a good grid selection will converge to a certain solution when the density is increased. We will explain the methodology of the chosen numerical panel methods used for calculating the aerodynamic characteristics of lifting surfaces (wing, horizontal tail) using VLM, and non lifting surfaces (body, vertical tail) using source-sink distribution. Finally, we will explain the methodology of calculation of the aerodynamic characteristics of complete airplane configuration in steady subsonic flow using the previously mentioned panel methods.

Computational Solution of The Lifting Surfaces

A well known method is described here for the numerical solution of the aerodynamic characteristics of lifting surfaces in incompressible flow. The VLM [14] represents the wing as a planner surface on which a grid of horseshoe vortices is superimposed (fig. 2). The velocity induced by each horseshoe vortex at a specified control point is calculated using the law of Biot-Savart (1). A summation is performed for all control points on the wing to produce a set of linear algebraic equations for the horseshoe vortex strengths that satisfies the boundary condition of no flow through the surface.

$$
\overline{\mathrm{dV}}=\frac{\Gamma_{\mathrm{n}}(\overline{\mathrm{dl}} \times \overline{\mathrm{r}})}{4 \pi \mathrm{r}^{3}}
$$

Application of the boundary condition that the flow is tangent to the wing surface at the control point of each of the $2 \mathrm{~N}$ panels provides a set of simultaneous linear algebraic equations (2) in the unknown vortex strength. When this set of equations has been solved the vortex strength distribution is known, hence the flow velocity is calculated and so pressure distribution and aerodynamic characteristics. $\left[\mathrm{a}_{\text {in }}\right]\left[\Gamma_{\mathrm{n}}\right]=-U_{\infty} \cdot\left[\mathrm{n}_{\mathrm{i}}\right]$

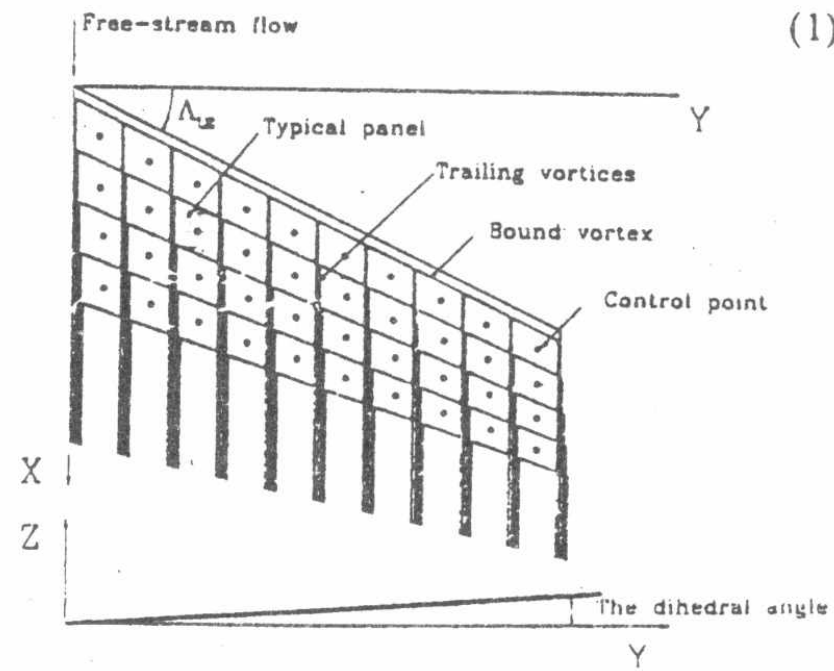

Fig. 2. Coordinate system, elemental panels, and horseshoe vortices for a typical wing planform in the VLM.

A possible solution of the lifting problem stated above is derived in refs. [2], [5], [14], [20]. Computational solution of the body

A general method described for the computational solution of the incompressible potential flow about three dimensional bodies (fig. 4). The method utilizes a source density distribution on the surface of the body and solves for the distribution necessary to make the normal component of fluid velocity as zero on the boundary. Plan quadrilateral surface elements are used to approximate the body surface (fig. 3), and the integral equation for the source density is replaced by a set of linear algebraic equations for the values of the source density on the quadrilateral elements. When this set of equations has been solved, the source density 
distribution is known, hence the flow velocity on the body surface is calculated. The problem considered is that of the steady flow of the perfect fluid about a three-dimensional body.

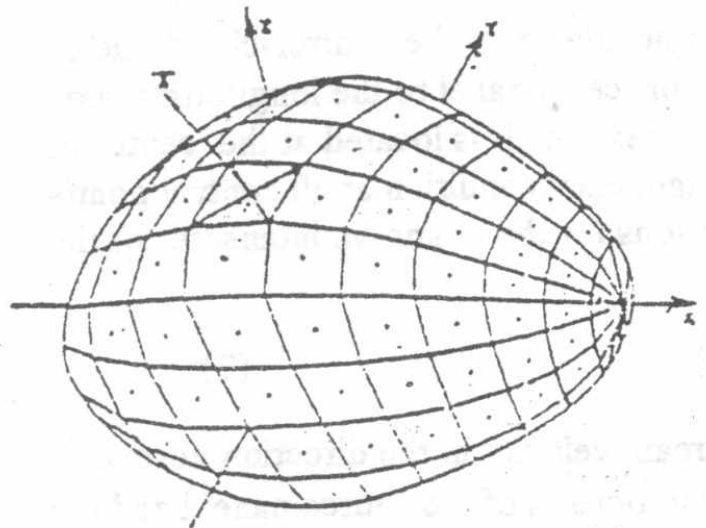

Fig. 3. Approximation of the body surface.

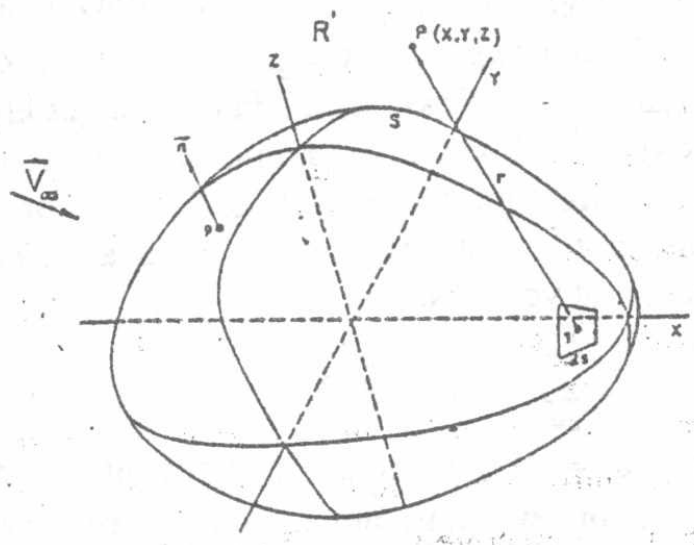

Fig. 4. Three dimensional body surface.

$\left[\mathrm{h}_{\mathrm{i}}\right]\left[\sigma_{1}\right]=-\mathrm{U}_{\cdot \infty} \cdot\left[\mathrm{n}_{\mathrm{i}}\right]$

Once the values of the source density on the quadrilateral elements have been obtained, the fluid velocities at points away from the body surface may also be calculated refs. $[2],[3],[4],[20]$. The total induced velocity at the $\mathrm{i}^{\text {th }}$ control point is $\mathrm{V}_{\mathrm{i}}$, the total velocity at that point is $\bar{V}_{i_{2}}$ and now the pressure coefficient can be calculated

$$
\begin{aligned}
& \bar{V}_{i}=\sum_{i=1}^{2 N_{i}} \bar{h}_{i l} \sigma_{1} \\
& \bar{V}_{i_{i}}=\bar{V}_{i}+\bar{U}_{\infty} \\
& C_{p_{i}}=1-\frac{V_{i_{i}}^{2}}{U_{\infty}^{2}}
\end{aligned}
$$

\section{Computational solution of wing-body-tail configuration:}

The main purpose of the present work is to develop a numerical method that could be used by aeronautical engineers as a tool for the preliminary design stage. Therefore, the method has to be sufficiently direct, simple to use, and efficient in order to be of practical engineering value. It was decided to simulate the body by a conventional source-panel method. Wings and all other lifting surfaces will be simulated by the VLM. It was assumed that the interference of wing-body-tail combinations can be obtained by simultaneous solution of the two systems solving for all velocities induced by each one on the other.

A numerical method for the calculation of the longitudinal aerodynamic characteristics of complete airplane configuration in steady subsonic flow has been developed. The method is based on a combination of the VLM for the lifting surfaces and source-panel method for the body. Special emphasis is given to the understanding of the behavior and the computational accuracy of the numerical method. In order to demonstrate the capabilities of the present method, distributed and total loads are computed and compared with available experimental results. A wing-body-Tail configuration is positioned in a uniform flow field of an undisturbed velocity vector $U_{\infty}$. The flow is assumed to be steady, incompressible, inviscd, and irrotional except for isolated vortices.

The body surface is divided into a number $\left(\mathrm{N}_{\mathrm{s}}\right)$ of trapezoidal panels, each supporting a source distribution of uniform intensity $\sigma$. A control point, at which the tangency boundary condition 
is satisfied, is located at the center of the panel area. The lifting surfaces are divided into a number $\left(\mathrm{N}_{\mathrm{k}}\right)$ of trapezoidal panels. Each panel has a horseshoe vortex system of strength $\left(\Gamma_{\mathrm{a}}\right.$ ), which consists of a straight bound vortex segment coincides with the quarter-chord line of the panel and of two semi infinite straight line trailing vortices parallel to the longitudinal axis ( $x$-axis). The boundary condition control point on the vortex panels is located at the center of the cell three-quarter chord lime. The application of the tangency condition at all control points results in the following system of linear algebraic equations for the unknown intensities of the sources and vortices:

$\sum_{i=1}^{N_{s}} h_{i l} \sigma_{1}+\sum_{n=1}^{N_{k}} a_{i n} \Gamma_{n}+b_{i}=0 \quad\left(i=1, \ldots, N_{s}+N_{k}\right)$

Where $b_{i}=\left(\bar{U}_{\infty} \cdot \bar{n}_{i}\right)$ is the component of the free stream velocity in the direction normal to panel $k$ control point, and $a_{\text {in }}$ and $h_{i l}$ are the influence coefficients of the source panel $l$ and the vortex $\mathrm{n}$, respectively, on the control point $\mathrm{k}$. The detail formulas of the influence coefficient are given in refs. [2],[3],[14],[20]. The solution of equation (7) determines the strengths of the singular elements, which are then used to compute the induced velocities at the $m^{\text {th }}$ control point. Knowing $\bar{U}_{\infty}$ we can get the total velocity at each panel $\bar{V}_{m_{l}}$, so the pressure coefficient and aerodynamic load at the $\mathrm{m}^{\text {th }}$ control point are

$\mathrm{C}_{\mathrm{p}_{\mathrm{m}}}=1-\frac{\mathrm{V}_{\mathrm{m}_{\mathrm{t}}}^{2}}{\mathrm{U}_{\infty}^{2}}$

$\overline{\mathrm{F}}_{\mathrm{m}}=-\mathrm{C}_{\mathrm{p}_{\mathrm{m}}}\left(0.5 \rho \mathrm{U}_{\infty}^{2}\right) \mathrm{s}_{\mathrm{m}} \overline{\mathrm{n}}_{\mathrm{m}}$

$\bar{F}_{t}=\sum_{m=1}^{\left(N_{k}+N_{w}\right)} F_{m}$

so we can get the lift coefficient from equation (10)

$\mathrm{L}_{\mathrm{t}}=\mathrm{F}_{\mathrm{t}_{\mathrm{z}}} \cos \alpha-\mathrm{F}_{\mathrm{t}_{\mathrm{x}}} \sin \alpha$

$\mathrm{C}_{\mathrm{t}_{\mathrm{t}}}=\mathrm{L}_{\mathrm{t}} /\left(0.5 \rho \mathrm{U}_{\infty}^{2}\right) \mathrm{S}$

Now, we can get total pitching moment about nose point of the aircraft by calculating the contribution of all the panels to that moment.

$M_{l e_{\mathrm{t}}}=\sum_{\mathrm{i}=1}^{\left(N_{\mathrm{k}}+N_{\mathrm{s}}\right)}\left(\mathrm{F}_{\mathrm{m}_{\mathrm{z}}} *\left(\mathrm{x}_{\mathrm{p}}-\mathrm{x}_{\mathrm{m}}\right)+\mathrm{F}_{\mathrm{m}_{\mathrm{x}}} *\left(\mathrm{z}_{\mathrm{n}}-\mathrm{z}_{\mathrm{m}}\right)\right)_{\mathrm{i}}$

where $\left(x_{n}, y_{n}, z_{n}\right)$ is the coordinates of the nose point, $\left(x_{m}, y_{m}, z_{m}\right)$ are the coordinates of the panel control point. The pitching moment coefficient is

$c_{\mathrm{m}_{\mathrm{le}},}=\mathrm{M}_{\mathrm{le}_{\mathrm{t}}} /\left(0.5 \rho \mathrm{U}_{\infty}^{2}\right) \mathrm{S} \mathrm{b}_{\mathrm{A}}$

where $\mathrm{S}$ is the wing area, $\mathrm{b}_{\mathrm{A}}$ is the mean aerodynamic chord

A vortex along the wing body intersection line is thus completely avoided. In the present work the vortex cells adjacent to the body are extended through the body up to the plane of symmetry. The velocities induced by the extended vortices are taken into account in the simultaneous solution of equation (7), but the tangency condition is not enforced on the exterded panels [6].

The proposed mathematical model for calculating longitudinal aerodynamic characteristics of different flight surfaces and their combinations, described in the previous section is applied to a case study of L-29, which is a jet trainer in the Egyptian air force. The computer programs in this work consists of two fully separated groups; (fig. 4) :

- Paneling programs group, used for dividing the individual surfaces of the configuration into a quadrilateral panels with four comer points. These programs were written in C-Language. For 
the purpose of the graphical verification of the paneled surfaces, we get the DXF files from the formatted output data files of the paneling programs and reading it in Autocad to check up the paneled shape.

- Calculation programs group, which takes the geometrical data of panels representing certain configuration from the paneling group, using it in calculating pressure distribution and the aerodynamic characteristics of the configuration. The calculation group of programs were written in Borland $\mathrm{C}+$ for windows. We can use these programs to solve the following problems:

- Solution of isolated lifting surfaces.

- Solution of multi-lifting surfaces.

- Solution of isolated body.

- Solution of wing-body combination. including the interference effects.

\section{Discussion Of Results}

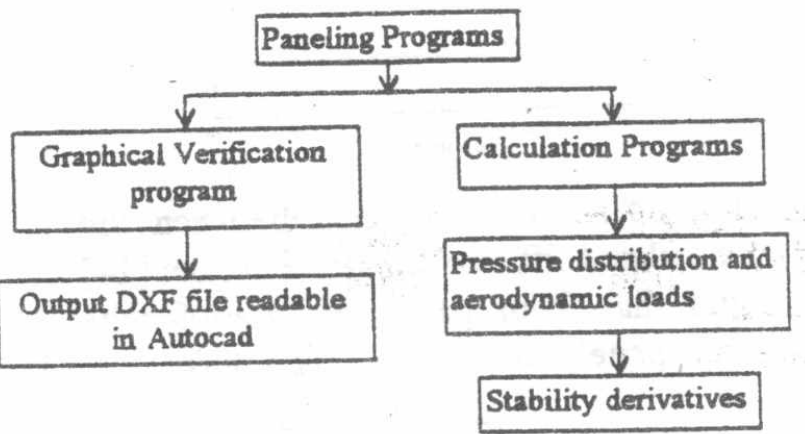

In this section the computational results are presented, analyzed and compared for many application cases. So this section consists of

- Lifting Surfaces Solution

- Nonlifting surfaces Solution

- Solution of wing-body-tail configuration.

\section{Lifting Surfaces Solution :}

Here, we will first verify our work by solving a previously solved example and comparing the results. Then we solve the isolated wing, isolated tail of the selected aircraft and getting the pressure coefficient distribution for every case at different angles of attack and different number of panels.

A. Verification :

To verify our work we solve example 7.2 , page 272 , ref. [14] of the four panel representation of a swept planner wing (fig. 5), taper ratio of unity, aspect ratio $=5, \Lambda=45^{\circ}$ (fig. 6.) introduces $A$ comparison between the calculated lift

Fig. 4. Computer Programs Tree

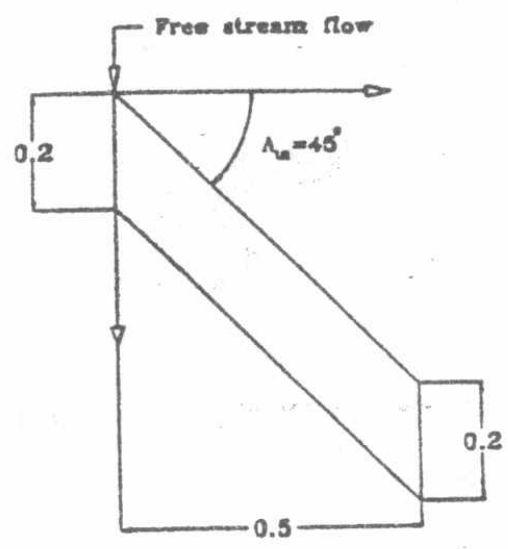

Fig. 5. Swept planner wing taper ratio of unity, $A R=5, \Lambda=45^{\circ}$. curve of the four panel swept wing from ref. [14] and that from partner program. Using the partner program we calculate the lift curves of the swept planner wing (dihedral angle $=0$ ) shown in (fig. 8). The convergence of the lift coefficient with number of panels for different angles of attack is shown in (fig. 9). The lift curves of (fig. 7) are calculated for a different number of panels of (fig. 8). In (fig. 7) the calculated lift curves are compared with the experimental lift curve of the swept planner wing of (fig. 5), where this experimental lift curve is taken from ref. [14].

B. Isolated Wing Solution :

Here, we are calculating the pressure coefficient distribution along isolated wing span of the selected aircraft model for different angles of attack and different number of panels. Then getting the convergence curves of the lift coefficient with number of panels for different 


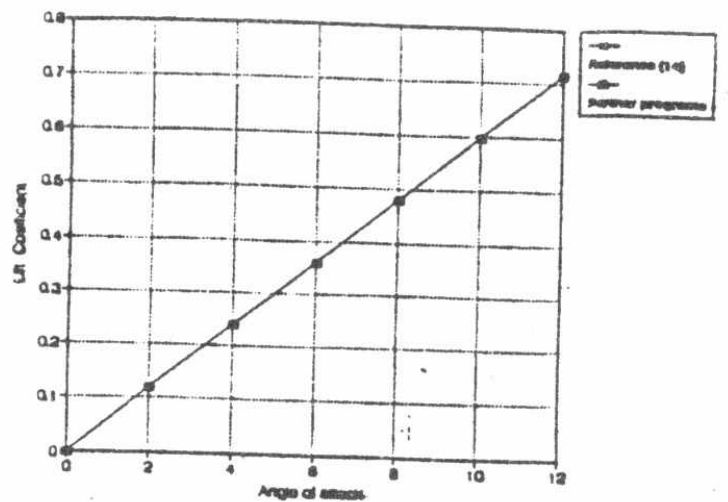

Fig. 6. A comparison between the calculated lift curve from reference [14] and that from partner program for the four panel swept wing of (fig. 8a).
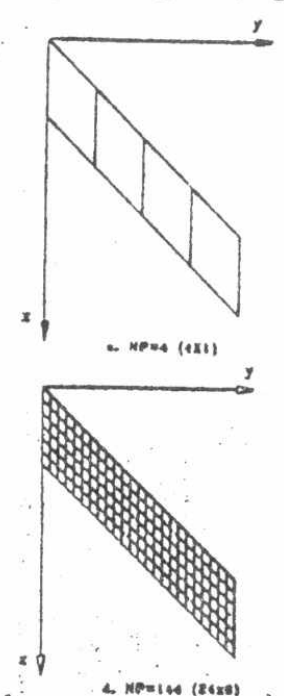

Fig. 8. Panel representation of the swept planner wing
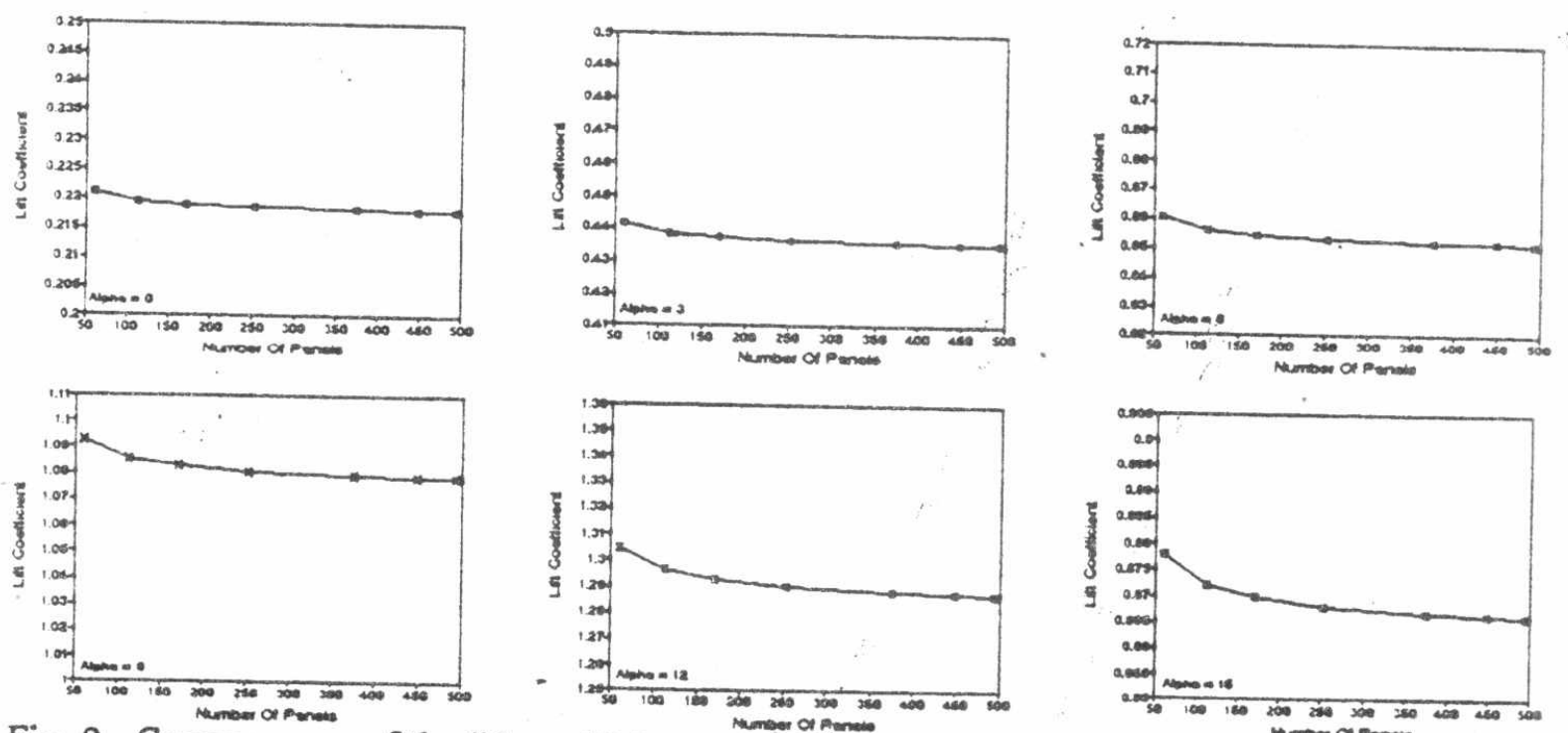

Fig. 9. Convergence of the lift coefficient with number of panels at different angles of attack. 
angles of attack. The graphical verification of the output data files from wing paneling program is shown in (fig. 10). (Fig. 11) represent the pressure coefficient difference distribution along half wing span for different number of panels $(N P=60, N P=170, N P=375, N P=493)$. of figs. (9a), (9c), (9e), (10f), these pressure coefficient difference distributions are made all over range of angles of attack changes from zero degree to fifteen degree with a step of three. From (fig. 11) we can notice the following:

1- The pressure coefficient difference increases as number of panels increases and that because the panel area decreases.

2- The curves have the same shape but the values are different.

3- A drop in the pressure coefficient difference value appears in the area between the first and the second trapezoidal of the selected aircraft wing. This drop appears due to the sudden change in the geometry between the first trapezoidal and the second one. To avoid this drop we increase the total number of panels on the wing camber surface. Also increasing the number of panels in that area where the sudden change appears. These solutions to avoid the sudden change and make it gradually change. But here we are limited with the computer capacity. The convergence curves for the lift coefficient with number of panels at different angles of attack are shown in (fig. 12).
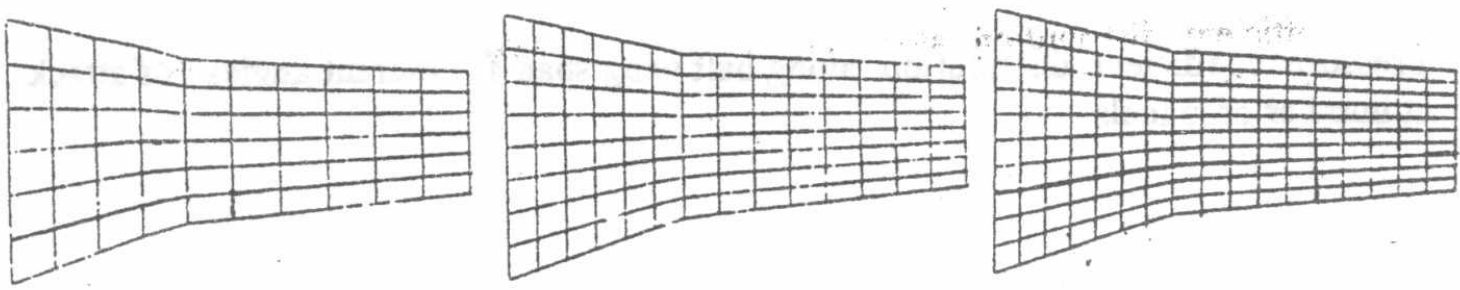

a. $N P W=60$

b. $N P W=112$

c. $N P W=170$
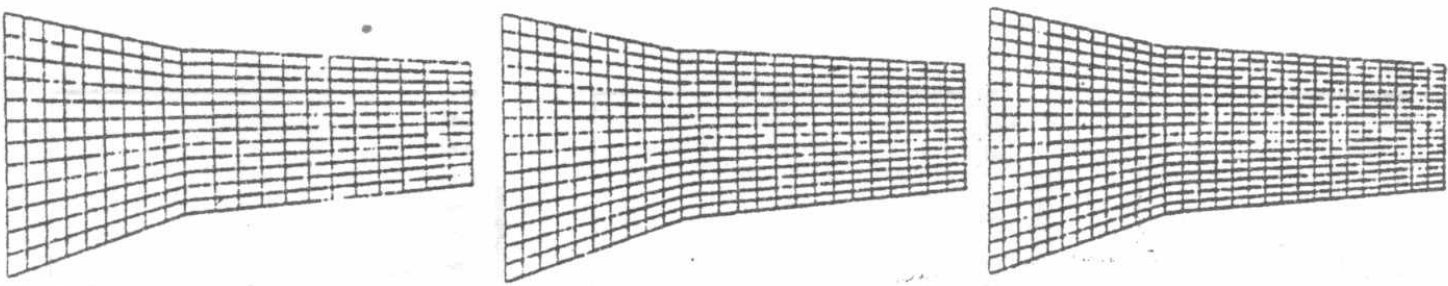

d. $N P W=252$

e. $N P W=375$

C. $N P H=493$

Fig. 10. Panel representation of the selected aircraft wiing

NonLifting Surfaces Solution :

In this section we are calculating the pressure coefficient distribution along the selected aircraft body. First, as a verification of our work (fig. 14) represents a comparison of the pressure coefficient distribution over a sphere surface along longitudinal axis, calculated using the potential equation for pressure coefficient eq. (14) [15] and that calculated using parner program. The graphical verification of the paneling technique of a sphere is shown in (fig. 13). $C_{p}=1-\frac{9}{4} \sin ^{2} \theta$

Now, we are calculating the pressure coefficient distribution over L-29 body surface. The graphical verification of the paneled L-29 body is shown in (fig. 15). The pressure coefficient distribution over L-29 body surface along body longitudinal axis for different body strips and at zero angle of attack is shown in (fig. 16.). 

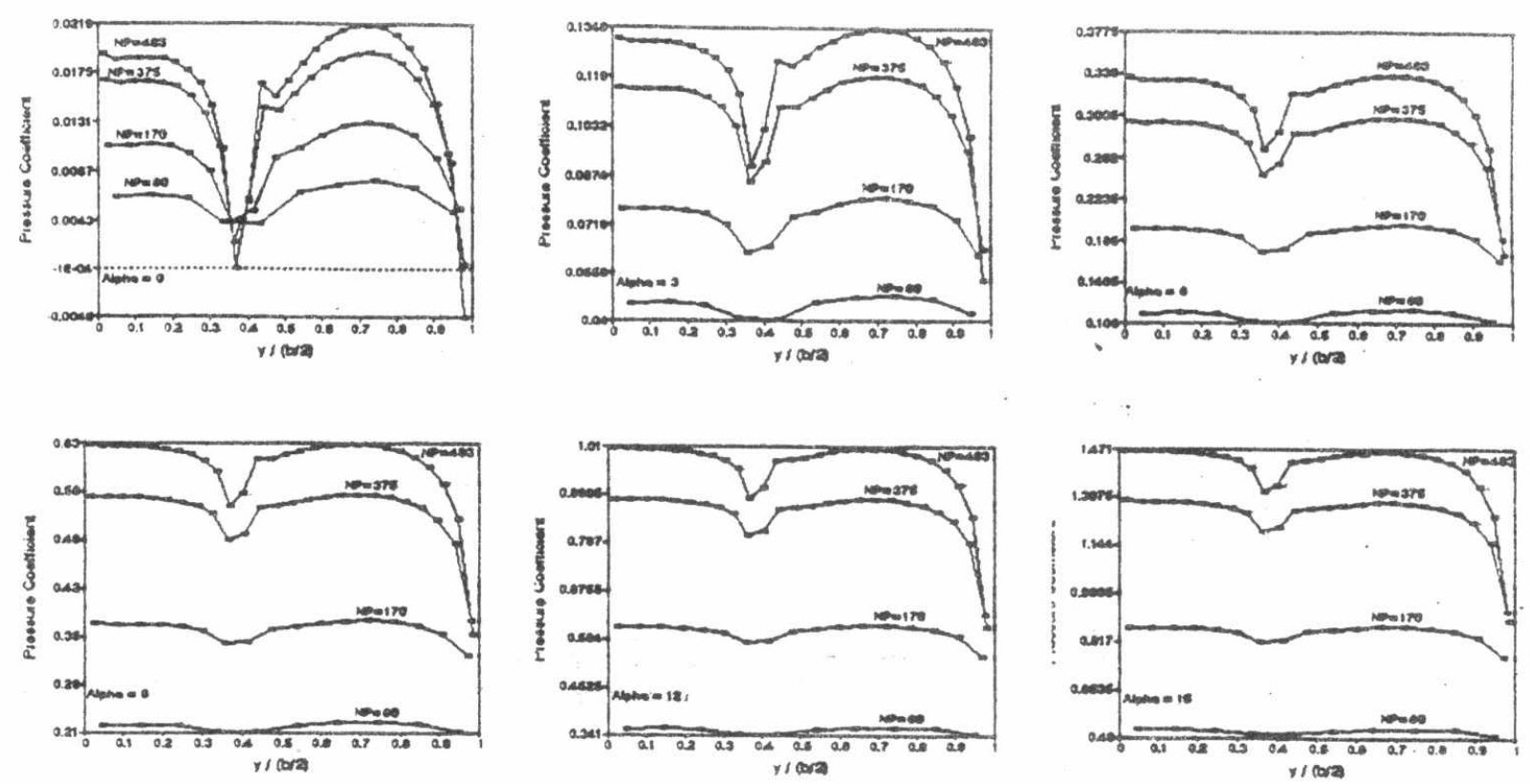

Fig. 11. Pressure coefficient distribution along half wing span at different angles of attack and different number of panels .
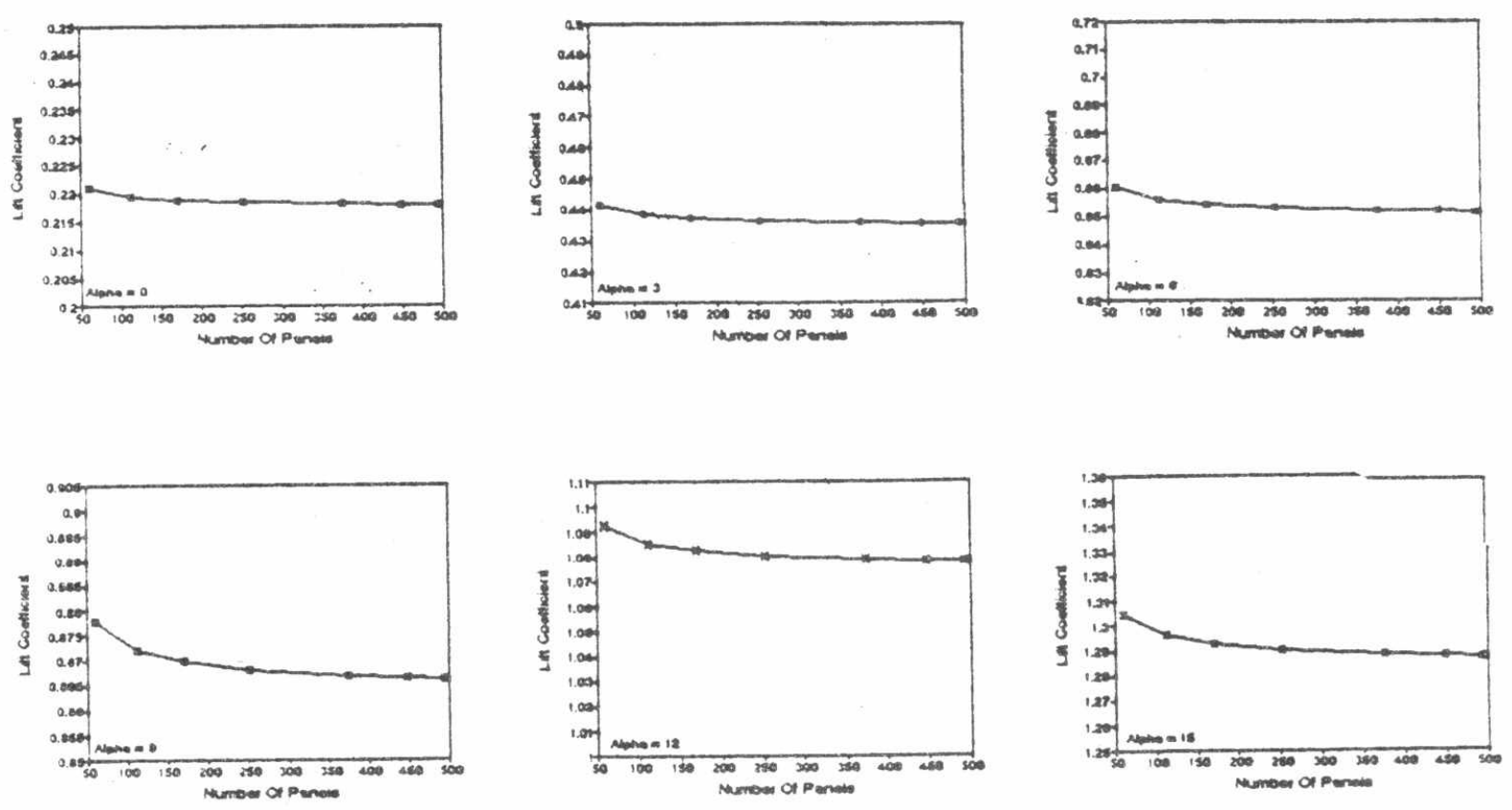

Fig. 12. Convergence of the lift coefficient with number of panels at different angles of attack. For the selected aircraft wing of (fig. 10) 


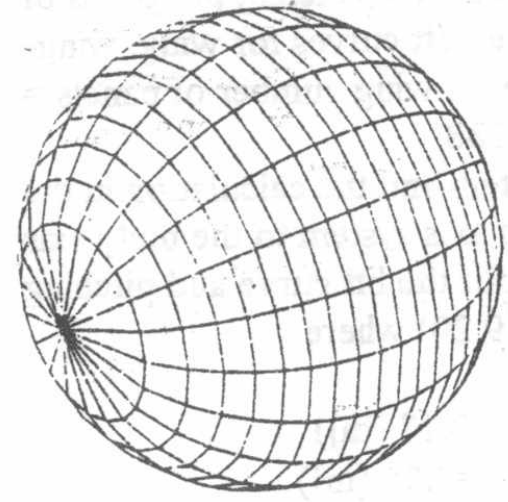

Fig. 13. Panel representation of a sphere, number of panels $=200$ (20*10)

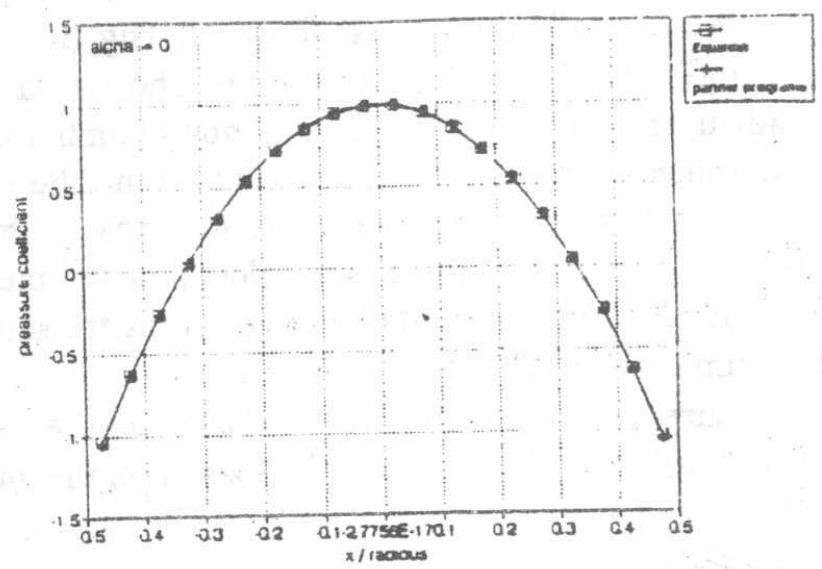

Fig. 14. Pressure coefficient distribution over sphere surface along longitudinal axis. Sphere number of panels $=200$, sphere radius $=0.5[\mathrm{~m}]$

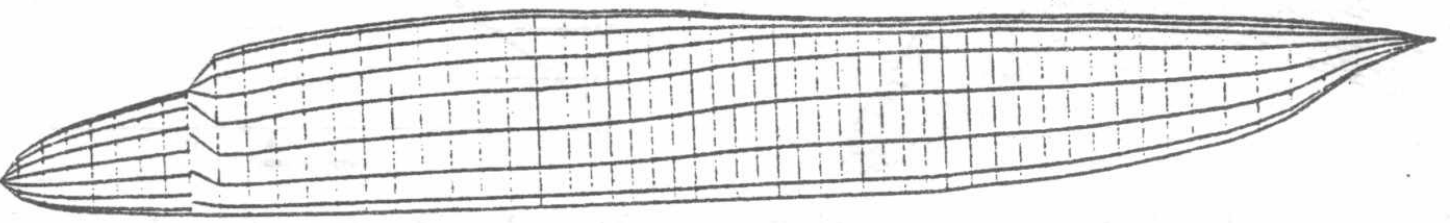

Fig. 15. Panel representation of L-29 body, number of panels $=448$
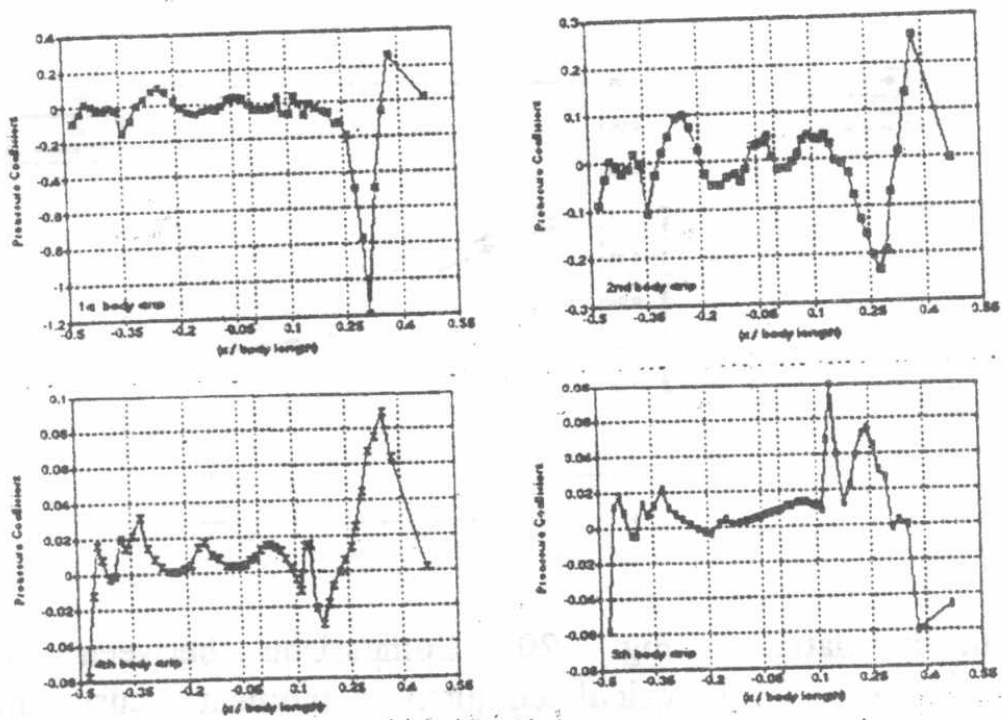

Fig. 16. Pressure coefficient distribution over body $\mathrm{BNP}=448$, angle of attack $=0$ [degree].
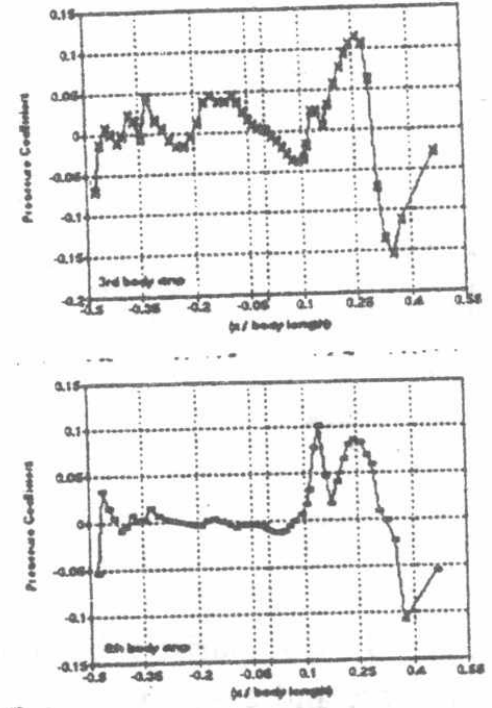
Solution of Wing-Body-Tail Configuration :

Our aim in this work is to solve wing-body-tail configuration to obtain some of the longitudinal characteristics in steady subsonic flow. So, first we verify graphically the paneling programs of wing-body-tail configuration as shown in (fig. 17). The calculated lift curves for wing, wingbody, wing-body-tail configurations are shown in (fig. 18) where: wing number of panels = 170 , tail number of panels $=42$ and body number of panels $=288$

The experimental measurements are made in the wind axis system and the calculation in the body axis system. So, we make a transformation from the wind axis system to the body axis system. Comparisons between the calculated and measured data for the lift curve and pitching moment curve of wing-body-tail configuration are shown in fig. $(19,20)$ where

- Mach number $=0.153$

$-\operatorname{Re}($ measurements $)=6 \times 10^{6}$

- $\operatorname{Re}($ calculation $)=7 \times 10^{7}$

(based on the model M.A.C. $=0.17[\mathrm{~m}]$ )

(based on the aircraft M.A.C. $=2.04[\mathrm{~m}]$ )

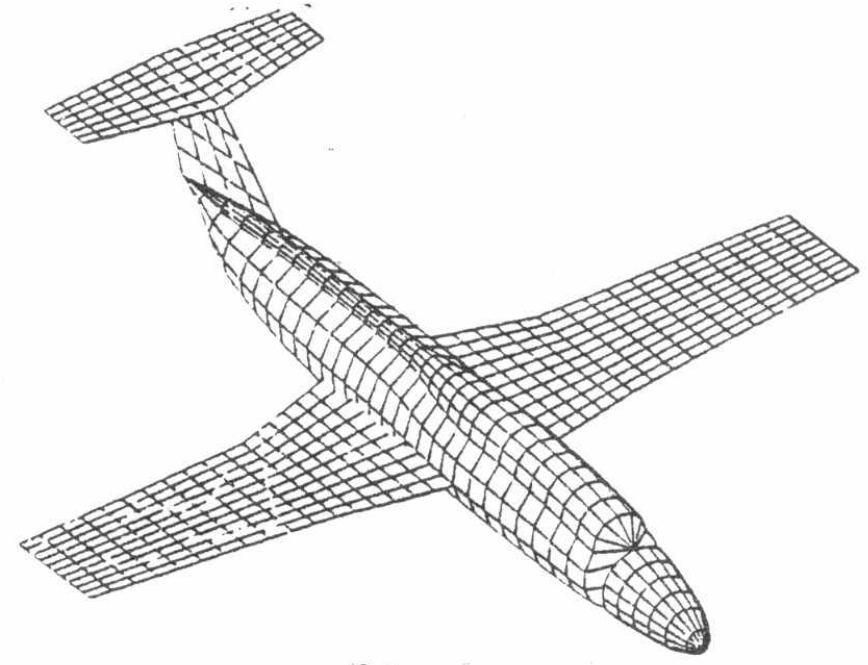

Fig. 17. Panel representation of the selected aircraft wing-body-tail configuration.

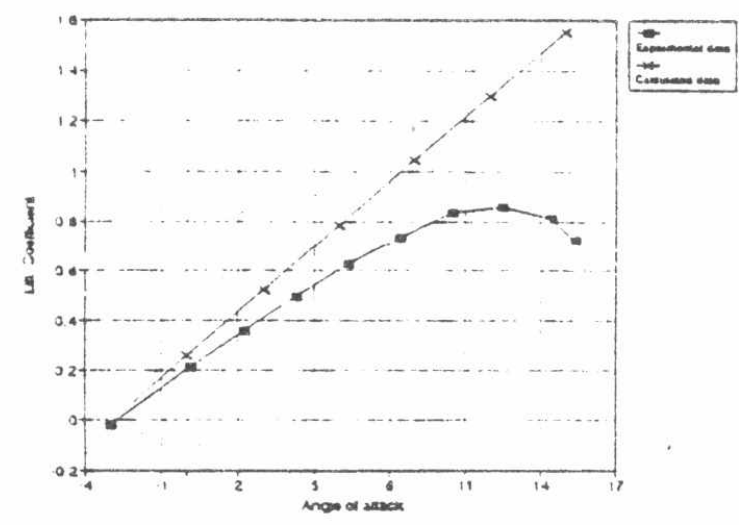

Fig. 19. Comparison between the calculated lift curve and experimental one.

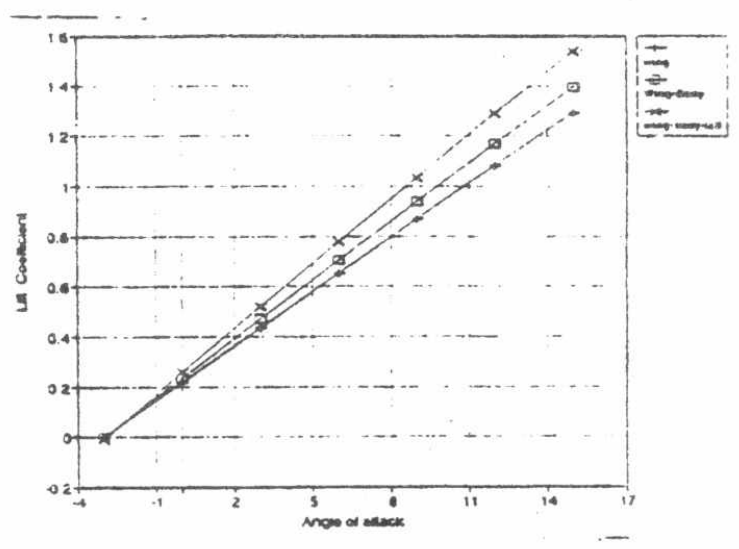

Fig. 18. Lift curves for wing, wingbody, wing-body-tail; $\mathrm{M}=0.153, \mathrm{Re}=$ $7 * 10^{\wedge} 7$

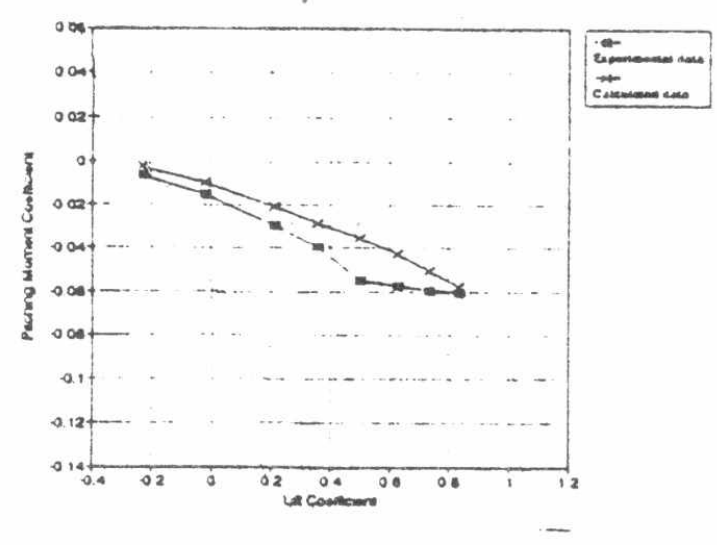

Fig. 20. Comparison between the calculated pitching moment curve and experimental one. 


\section{CONCLUSION AND FUTURE WORK}

Computational analysis has been considered as a powerful, economical and useful tool for determining the aerodynamic coefficients of a complete aircraft configurations. In the present work, three different cases have been investigated. They are solved using the same computer code, which has been developed in C-language. The configuration surfaces have been divided into panels through another computer code. These codes have been verified.

\section{CONCLUSIONS}

The investigated cases are:

Lifting surfaces solution. The surface has been divided into the required number of panels on the camber line and with the required distribution. The developed calculation code can compute the induced velocity distribution over the surface, so the pressure coefficient difference distribution on the surface, and the surface lift curve. The developed code is verified by solving a previously solved example and comparing the results.

- Nonltilifting surface solution. The body has been divided into surface panels with four comer points and then graphically verified. The calculation programs calculate the source density distribution over the body surface, and then the pressure coefficient distribution over the surface.

- Solution of complete configuration. The calculation programs compute the source density distribution over the nonlifting surface, the vortex density distribution over the lifting surfaces, hence compute the induced velocity distribution over the configuration surface, and the pressure coefficient distribution taking into account the interference between the lifting surfaces and nonlifting surface. Finally, we get the lift curve and pitching moment curve for wing-body-tail configuration.

The following could be conchuded:

- The pressure coefficient distribution over the lifting surfaces increases as number of panels increases, tell we reach a number of panels that will simulate configuration.

- The panel methods are geometrical methods, and they are affected with the geometrical changes. So, the number of panels must be increased in areas with geometrical changes.

\section{FUTURE WORK}

The present work is a contribution to the calculation of the aerodynamic coefficients of an aircraft as a whole in a global system of equations, in steady linear incompressible invicid flow. As an extension of this.work, the following might be considered:

- Nonlinear solution of a lifting surfaces in Steady, incompressible, invicid flow.

- Nonlinear solution of a multilitting surfaces in Steady, incompressible, invicid flow.

- Unsteady solution of a complete aircraft configuration.

\section{LIST OF SYMBOLS}

$\begin{array}{ll}a_{i n} & \text { Influence coefficient (at } \Gamma=1 \text { ) of panel } n \text { on control point of panel m. } \\ C_{c_{1}} & \text { Lift coefficient. } \\ C_{t_{t}} & \text { Total lift coefficient. } \\ C_{m} & \text { Pitching moment coefficient. } \\ C_{p} & \text { Pressure coeficient difference. } \\ D_{t} & \text { Total drag force }[N] . \\ \overline{d V} & \text { Velocity induced by a vortex filament of strength } \Gamma_{0} \text { and length dl. }\end{array}$




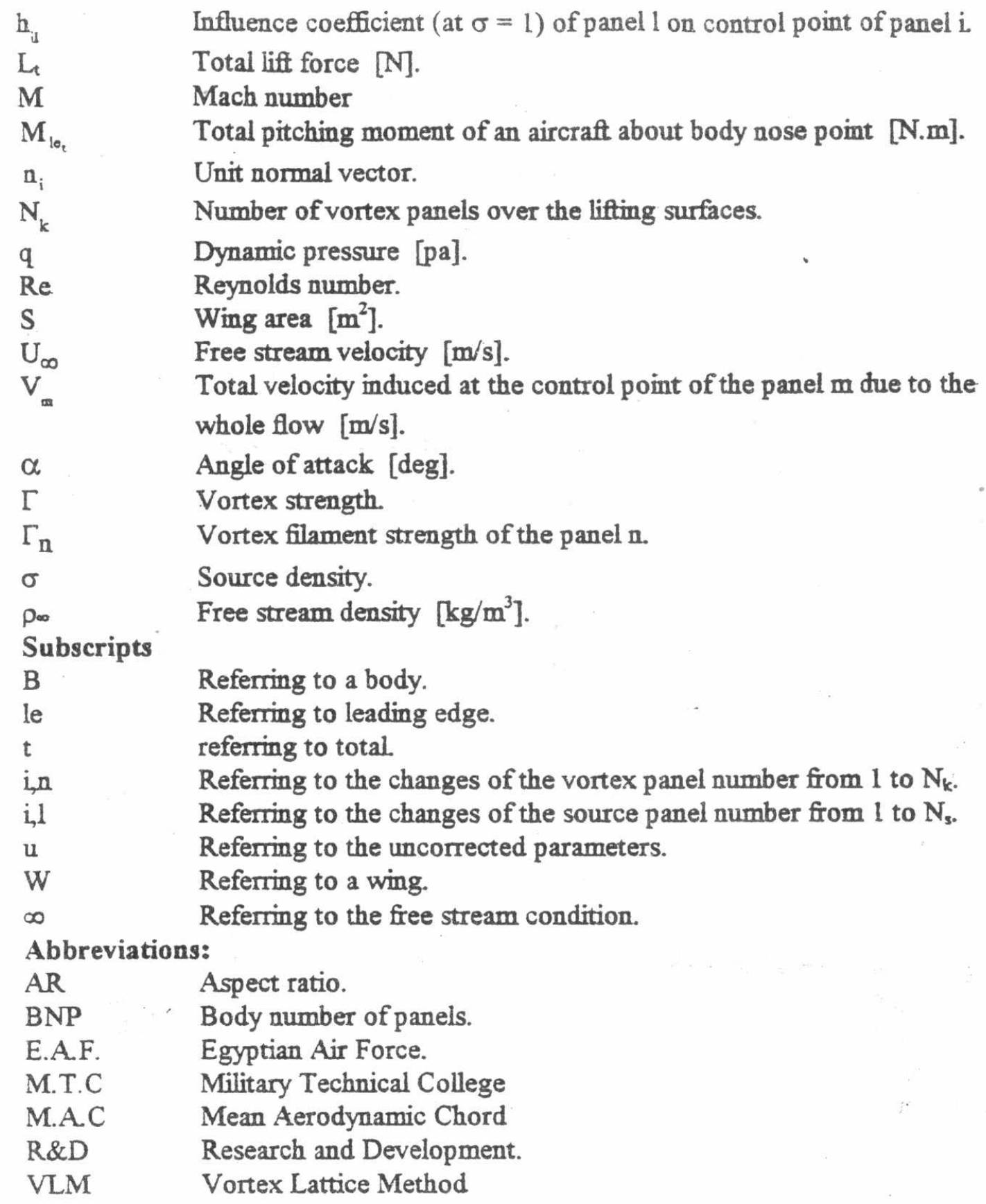

\section{REFERENCES}

1. Miranda, L. R, "Application of Computational Aerodynamics to Airplane design," Journal of Aircraft, Vol. 21, No. 6, June 1984, pp. 355-370.

2. Katz, J. and Plotken, A. "Low-Speed Aerodynamics From Wing Theory to Panel Methods," McGraw-Hill, 1991

3. J.L. Hess, and A.M.O. Smith, "Calculation of nonlifting potential flow about arbitrary three dimensional bodies," Douglas Aircraft company Report No. ES 40622, March 1962.

4. J.L. Hess, and A.M.O. Smith, "Calculation of nonlifting potential flow about arbitrary three dimensional bodies," Journal of Ship Research, Vol. 8, No. 2, September 1964, pp. 22-44 
5. Almosnino, D., Zorea, C., and Rom, J., "A Method for Calculating Longitudinal Characteristics of Wings and Multiple Lifting Surfaces in Subsonic Flow, and at High Angles of Attack," Israel Journal of Technology, Vol. 16, 1978, pp. 132-141

6. Rusak, Z., Wasserstorm, E., and Seginer, A., "Numerical Calculation of Nonlinear Aerodynamics of Wing - Body Configurations," AIAA Journal, Vol. 21, No. 7, July 1983, pp. 929-936.

7. Rusak, Z., "Numerical Calculation of Wing - Body Configurations," Msc Thesis, Technion--Israel institute of Technology, Haifa, Jan. 1982:

8. Asfar, K.R, Mook, D.T., and Nayfeh, A.H., "Application of Vortex Lattice Technique to Arbitrary Bodies," Joumal of Aircraft, Vol. 16, No. 7, July 1979, pp. 421424.

9. Roos, R., "The Use of Panel Methods For Stability Derivatives," Agard Conference, 1979.

10. Falkner, V.M.; "The Calculation of Aerodynamic Loading on Surfaces of any Shape", Report and Memoranda 1910, Aeronautical Research Committee, Aug. 1943.

11. Lan, C.E.; "A Quasi Vortex Lattice Method in thin wing Theory," Journal of Aircraft, Vol. 11, No. 9, Sept. 1974, PP. 518-527.

12. Roggero, F.and Larguier R, "Aerodynamic calculation of three dimensional configurations," Joumal of Aircraft, Vol. 30, No. 5, Spet. - Oct. 1993.

13. Cenko, A., "PAN Air Application to complex configurations," Journal of Aircraft, Vol. 20, No. 10, October 1983.

14. Bertin, J.J. and Smith, M.L., "Aerodynamics For Engineers," 2nd Edition, Prentic-Hall International, Inc., 1989.

15. Anderson, J.B., Jr., "Fundamentals of Aerodynamics," 2nd printing, McGraw-Hill Book company, Singapore, 1986.

16. Abbott, L.H. and Doenhoff, A.E.V., "Theory of wing sections," Dover Publications, Inc., New York, 1959.

17. Essawy, M.E., 'Dingler Wind Tunnel Aerodynamic Characteristics Determination," Entrance Interview Report, M.T.C., Cairo, 1969.

18. Katary, M.H., "Analysis of Jet-Trainer Airplane Longitudinal Stability," Entrance Interview Report, M.T.C., Cairo, 1974.

19. Pope, A. and Haper, J.J., 'Low-Speed Wind Tunnel Testing," Johen Wiley \& Sons, Inc., 1966.

20. Mostafa, M. I.I., "Computational Solution of Aircraft Stability Derivative," Msc Thesis, M.T.C., Cairo, 1995. 